\title{
Enhanced iNOS Expression in Leukocytes and Circulating Endothelial Cells Is Associated with the Progression of Coronary Artery Lesions in Acute Kawasaki Disease
}

\author{
XIANYI YU, KEI-ICH HIRONO, FUKIKO ICHIDA, KEI-ICHIRO UESE, CHEN RUI, \\ SAYAKA WATANABE, KAZUHIRO WATANABE, IKUO HASHIMOTO, TOKIMASA KUMADA, \\ EIKICHI OKADA, MASARU TERAI, ATSUKO SUZUKI, AND TOSHIO MIYAWAKI \\ Department of Pediatrics [X.Y., K.H., F.I., K.U., C.R., S.W., K.W., I.H., T.M], Department of Pathology-1[ \\ T.K., E.O.], Toyama Medical and Pharmaceutical University, 930-0194 Toyama, Japan, Department of \\ Pediatrics, Chiba University, 260-8670 Chiba, Japan [M.T.], and Department of Pediatrics, Tokyo Teishin \\ Hospital, 102-8796 Tokyo, Japan [A.S.]
}

\begin{abstract}
ABST
Nitric oxide (NO) serves many vasoprotective roles, but the
massive release of NO causes arterial wall degeneration. We
investigated whether enhanced nitric oxide synthase (iNOS)
expression in peripheral blood leukocytes and circulating endo-
thelial cells mirrors the progression of coronary arterial lesions in
55 children with acute Kawasaki disease (KD), including 24 with
and 31 without coronary artery lesions $(\mathrm{CAL})$. Patients were
treated with i.v. gamma-globulin at the time of diagnosis and
blood samples were collected before and after treatment. The
cellular origin of NO synthesis was determined by flow cytomet-
ric analysis of iNOS expression in peripheral blood, and by
immunohistochemical analysis of circulating endothelial cells
and coronary arteries. iNOS expression in neutrophils peaked at
the time of diagnosis, but did not peak in monocytes until 2 wk
post onset of disease. Levels were significantly higher in both
cell types in patients with CAL ( $p=0.001$ and $p=0.035$,
respectively). In addition, the number of circulating endothelial
cells and levels of iNOS expression were higher in patients with
CAL ( $p=0.011$ and $p=0.012$, respectively). Immunohisto-
\end{abstract}
chemical analysis of the coronary arteries from three patients with acute KD revealed iNOS immunoreactivity in endothelial cells, as well as infiltrating monocytes/macrophages in the aneurysms. We conclude that the expression of iNOS in peripheral blood leukocytes, as well as circulating endothelial cells, correlates with the severity of coronary arterial wall injury and the progression of CAL in patients with acute KD. (Pediatr Res 55: 688-694, 2004)

$\quad$ Abbreviations
KD, Kawasaki disease
CAL, coronary artery lesions
VEGF, vascular endothelial growth factor
NO, nitric oxide
eNOS, endothelial nitric oxide synthase
iNOS, inducible nitric oxide synthase
IVGG, i.v. gamma-globulin
DMFI, differences of the mean fluorescence intensity

$\mathrm{KD}$ is an acute systemic vasculitis syndrome primarily affecting small and medium-sized arteries, with the development of severe CAL occurring in $10-15 \%$ of affected children (1-3). Although KD is the primary cause of acquired heart disease in children in Japan and the United States, the etiology and mechanisms of CAL development remain unclear. Histopathologic studies have shown that early coronary vascular

Received March 20, 2002; accepted December 1, 2003

Correspondence: Fukiko Ichida, M.D., Department of Pediatrics, Toyama Medical and Pharmaceutical University, 2630 Sugitani, Toyama, Toyama, 930-0194 Japan; e-mail: fukiko@ms.toyama-mpu.ac.jp

X.Y. is supported by grants from the Ministry of Education of China. K.U. is supported by grants from Japan Foundation of Cardiovascular Research.

DOI: 10.1203/01.PDR.0000113464.93042.A4 lesions of KD are characterized by infiltration of large numbers of mononuclear cells, such as monocytes/macrophages and lymphocytes, suggesting these cells play a key role in the progression of CAL (4-7). Supporting this hypothesis, we have previously reported that VEGF derived from peripheral blood mononuclear cells might contribute to vascular injury and remodeling in acute $\mathrm{KD}(8)$. In vitro studies have shown that VEGF increases NO release in human endothelial cells (9) and modulates microvascular permeability via a signaling cascade involving NO synthesis in coronary venules (10).

NO, which is synthesized by two isoforms of nitric oxide synthase termed endothelial (eNOS) and inducible (iNOS), plays diverse roles in the physiology and pathophysiology of the cardiovascular system (11-13). NO is a signaling molecule 
in blood vessels, where the release of NO from endothelial cells, synthesized by eNOS, acts on the underlying smooth muscle to maintain vasodilation. After arterial injury, NO serves a number of vasoprotective roles, including inhibition of platelet aggregation and leukocyte adherence, and vascular smooth muscle cell proliferation. In contrast, the release of large amounts of NO, primarily synthesized by iNOS in macrophages, neutrophils, endothelial cells, and smooth muscle cells, from the cardiovascular wall in response to inflammatory stimuli causes arterial wall degeneration and shock $(14,15)$. A recent study elucidated that $\mathrm{NO}$ synthesis is a key requirement for cerebral aneurysm formation, which can be prevented by inhibition of iNOS (16).

Therefore, we hypothesized that leukocytes, as well as endothelial cells, actively generate NO by expression of iNOS, and that enhanced iNOS expression in peripheral blood leukocytes and circulating endothelial cells might correlate with the development of CAL during acute KD. To investigate this hypothesis, we determined the cellular origin of NO by flow cytometric analysis of iNOS expression in peripheral blood leukocytes and by immunohistochemical analysis of circulating endothelial cells and coronary arteries.

\section{METHODS}

Study population and blood samples. Fifty-five patients (26 males and 29 females), aged 4 mo to 7 y 3 mo $(2.4 \pm 1.7$ y), with acute KD were enrolled between October 1999 and September 2001. The patients were further classified as those with $(n=24)$ or without CAL $(n=31)$ (Table 1). Fifteen normal controls were also enrolled. The patients and controls were seen at the university hospital or at one of nine affiliated hospitals. All KD patients fulfilled the diagnostic criteria established by the KD research committee (17) and were treated with IVGG at $400 \mathrm{mg} / \mathrm{kg}$ for $5 \mathrm{~d}$ as well as oral aspirin (30 $\mathrm{mg} / \mathrm{kg} / \mathrm{d}$ ). Two-dimensional echocardiography was performed before and after treatment with IVGG and at 2, 3, and 4 wk after the onset of $\mathrm{KD}$. The time of disease onset was defined as the day on which fever developed. A coronary artery with a diameter of $3 \mathrm{~mm}$ or more (4 $\mathrm{mm}$ if the subject was over the age of $5 \mathrm{y}$ ) by echocardiogram was defined as abnormal according to the diagnostic criteria of the Research Committee on KD (17). Parental informed consent was obtained for each child enrolled in this study. The Research Ethics Committee of Toyama Medical and Pharmaceutical University Hospital approved the study.

Blood samples were collected at the time of diagnosis before the initiation of IVGG treatment, immediately after the first
IVGG injection, and then at 2,3, and 4 wk after disease onset. Fresh heparinized venous blood was used for flow cytometric analysis and quantitative studies of endothelial cells, and samples collected between October 1998 and September 2000. Blood samples were collected from healthy controls on a single occasion during the same period.

In addition, specimens of coronary arteries were obtained from three patients who died of acute fatal KD (Table 2) and from a trauma victim control who had no evidence of cardiovascular disease.

Flow cytometric assay of iNOS expression. Neutrophils and mononuclear cells were isolated from heparinized venous blood by dextran sedimentation and Ficoll-Hypaque gradient centrifugation, as previously described (18). Two-color immunofluorescence analysis of iNOS expression in mononuclear cell subpopulations was performed as previously described (18). In brief, to discriminate between monocytes and lymphocytes, mononuclear cells were stained with phycoerythrin (PE)-labeled CD14 MoAb (IgG2a; DAKO Japan, Kyoto, Japan). iNOS expression was determined by staining with FITClabeled iNOS MoAb (Transduction Laboratories, Lexington, KY, U.S.A.). The stained cells were analyzed using a Cytoron Absolute flow cytometer (Ortho-Clinical Diagnostics, Tokyo, Japan). To quantify the data, the DMFI was defined as "differences of the mean fluorescence intensity" between a control antibody and the anti-iNOS antibody.

Quantitation of circulating endothelial cells. Circulating endothelial cells were quantitated by immunohistochemical staining of "buffy-coat" smears, as described by Solovey et al. (19). In brief, the "buffy-coat" layer, supernatant, and interface of heparinized venous blood separated by dextran sedimentation and Ficoll-Hypaque gradient centrifugation, as described above, were pooled and centrifuged for $5 \mathrm{~min}$ at $1200 \times \mathrm{g}$. After the removal of supernatant, the resulting "buffy-coat" pellet was resuspended in RPMI 1640 and transferred to six slides such that each slide would contain cells from the equivalent of $1 \mathrm{~mL}$ of whole blood. Smears were air-dried overnight and then fixed with $4 \%$ paraformaldehyde for $20 \mathrm{~min}$. To identify circulating endothelial cells, the cells were stained with the mouse anti-endothelial cell MAb, P1H12 (diluted to 2 $\mu \mathrm{g} / \mathrm{mL}$; Chemicon International, Temecula, CA, U.S.A.) (19). The cells were stained with a rabbit polyclonal antibody against mouse iNOS (diluted 1:50; Upstate Biotechnology, Lake Placid, NY, U.S.A.) and a mouse MAb against human eNOS (diluted 1:10; Transduction Laboratories) to identify whether circulating endothelial cells express iNOS and eNOS in acute KD. The other antibodies we used were APAAP

Table 1. Clinical laboratory data in acute KD with or without $C A L$

\begin{tabular}{lccc}
\hline & With CAL & Without CAL & $p$ Value \\
\hline No. of patients & 24 & 31 & \\
Age (y) & $2.1 \pm 1.9$ & $2.5 \pm 2.0$ & NS \\
Max. CRP $(\mathrm{mg} / \mathrm{dL})$ & $14.4 \pm 5.9$ & $9.2 \pm 4.6$ & 0.014 \\
Max. WBC $\left(10^{3} / \mathrm{mm}^{3}\right)$ (neutrophil) & $15.0 \pm 4.4(70 \pm 17)$ & $14.9 \pm 5.1(69 \pm 15)$ & $\mathrm{NS}$ \\
Duration of fever $(\mathrm{d})$ & $9.0 \pm 1.9$ & $6.3 \pm 1.3$ & 0.001 \\
Max. diameter of coronary artery $(\mathrm{mm})$ & $3.9 \pm 0.6$ & $2.2 \pm 0.3$ & $<0.001$ \\
\hline
\end{tabular}

Data are mean \pm SD. $p$ Value indicates difference between groups. CRP, C-reactive protein; WBC, white blood cells. 
Table 2. Characteristics of three patients who died of acute KD

\begin{tabular}{|c|c|c|c|c|c|c|}
\hline Patient & Age & Sex & $\begin{array}{c}\text { Time since } \\
\text { onset }\end{array}$ & $\begin{array}{c}\text { Cause of } \\
\text { death }\end{array}$ & CAL & Treatment \\
\hline 1 & $3 \mathrm{mo}$ & $\mathrm{F}$ & d 18 & AMI & Multiple An in RCA and LCA & IVGG and oral aspirin \\
\hline 2 & $4 \mathrm{y}$ & M & d 14 & Rupture of An & Giant An in LCA & Oral aspirin \\
\hline 3 & $2 \mathrm{mo}$ & M & d 15 & Rupture of An & Multiple giant An in RCA and LCA & IVGG and oral aspirin \\
\hline
\end{tabular}

AMI, acute myocardial infarction; An, aneurysm; RCA, right coronary artery; LCA, left coronary artery.

mouse monoclonal (DAKO, Glostrup, Denmark) and rabbit anti-mouse immunoglobulins (DAKO). The fast red substrate (Sigma Chemical, St. Louis, MO, U.S.A.) was applied for color development. The samples were visualized by light microscopy and only nucleated cells were counted.

Immunohistochemistry in coronary artery in acute KD. The specimens of coronary artery were fixed in formalin, dehydrated, embedded in paraffin blocks, cut into 4- $\mu \mathrm{m}$ sections, and mounted on slides. The sections were incubated with $0.3 \%$ hydrogen peroxide to abolish endogenous peroxidase activity, and then incubated with 5\% BSA in Tris- $\mathrm{HCl}$ buffer $(\mathrm{pH}$ 7.4) to block nonspecific immunohistochemical reactions. The sections were incubated overnight at $4^{\circ} \mathrm{C}$ with antibodies against iNOS (diluted 1: 500; Affinity Bioreagents, Golden, CO, U.S.A.), eNOS (diluted 1:100: Transduction Laboratories) (16), and CD68 (diluted 1:100: DAKO Japan). After washing, the sections stained with the anti-iNOS antibody were incubated with goat anti-rabbit immunoglobulin conjugated to peroxidase labeled-dextran polymer (EnVision+, peroxidase, rabbit: DAKO, Carpinteria, CA, U.S.A.) for $30 \mathrm{~min}$. The sections stained with anti-eNOS and anti-CD68 antibodies were incubated with rabbit anti-mouse immunoglobulin conjugated to peroxidase labeled-dextran polymer $($ EnVision + , peroxidase, mouse: DAKO) for $30 \mathrm{~min}$. After washing, the peroxidase activity was detected with $0.02 \% 3,3^{\prime}$-diaminobenzidine tetrahydrochloride containing $0.005 \%$ hydrogen peroxidase in 0.05
M Tris-HCL buffer ( $\mathrm{pH}$ 7.4). The slides were counterstained with hematoxylin and eosin (H\&E) and mounted.

Statistics. Results were expressed as mean \pm SD. Analysis of the differences between groups of patients was accomplished using ANOVA followed by Dunnett's multiple comparison. The difference was considered significant with a $p$ value $<0.05$.

\section{RESULTS}

Patient characteristics and clinical laboratory data. Table 1 shows the clinical characteristics of the 55 acute KD patients enrolled, comparing patients with CAL and those without. All patients were diagnosed and began treatment between 4 and $7 \mathrm{~d}$ after disease onset. Patients with CAL had significantly higher maximum concentrations of C-reactive protein and longer durations of fever than those without CAL. There were no significant differences between groups with respect to age and the maximum number of white blood cells (WBC). Maximal WBC counts were observed before IVGG treatment and approximately $70 \%$ of the WBC were neutrophils in both groups. The diameter of the coronary arteries in KD patients with CAL was observed to be maximal at $10-16 \mathrm{~d}$ (mean, $13.9 \pm 1.1 \mathrm{~d}$ ) after onset. Four of the 24 patients with CAL developed coronary aneurysms, which were verified by selective coronary angiography in the convalescent phase.
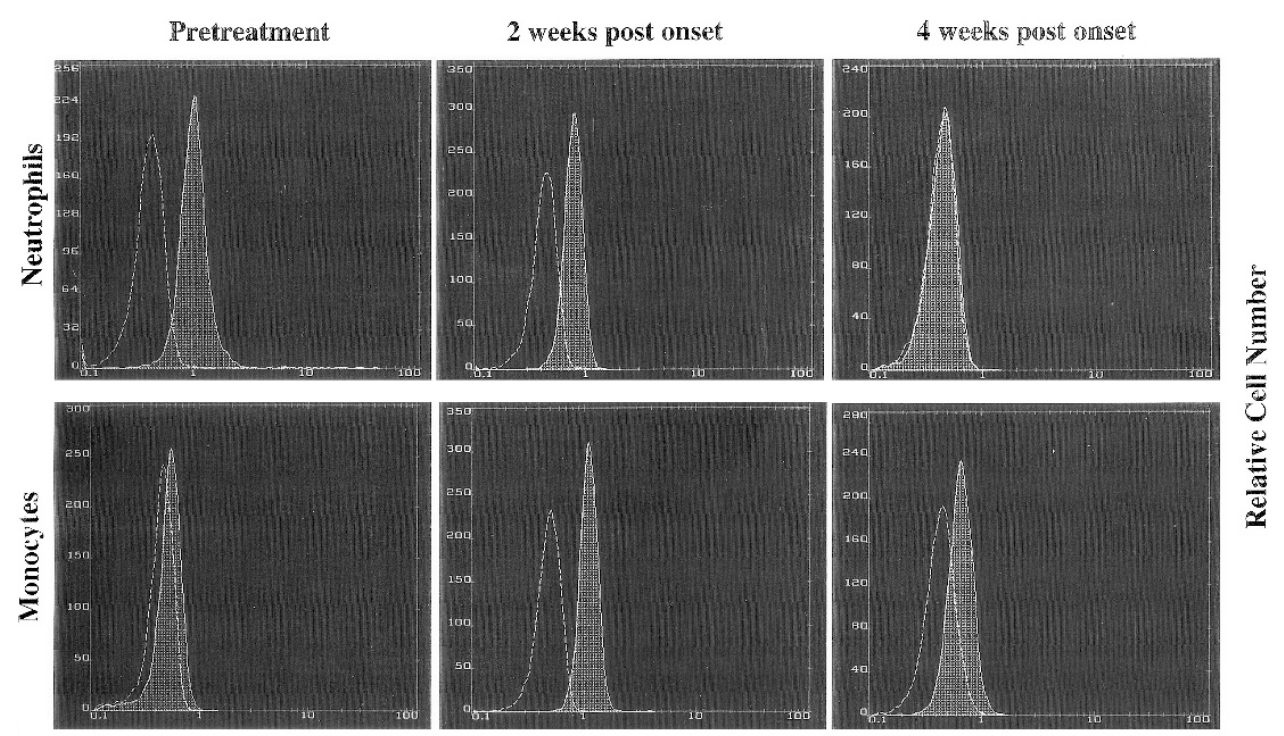

\section{Fluorescence Intensity}

Figure 1. Flow cytometric analysis of intracellular iNOS expression in neutrophils and monocytes from a representative KD patient with CAL. The dotted and solid lines represent the staining with control antibody and anti-iNOS antibody, respectively. 
iNOS expression in neutrophils and monocytes. Evaluation of iNOS expression identified different expression patterns in neutrophils and monocytes (Fig. 1). Neutrophils strongly expressed iNOS during the early stage of acute KD, and levels were significantly higher at the time of diagnosis than in controls (DMFI, $0.86 \pm 1.1$ versus $0.15 \pm 0.23, p=0.023$ ) but were normal at later time points. In contrast, iNOS expression in monocytes peaked at $2 \mathrm{wk}$ after disease onset with expression continuing even at the 4-wk evaluation. iNOS expression in monocytes was significantly higher in the KD patients than in controls at $2 \mathrm{wk}$ (DMFI, $0.87 \pm 0.35$ versus $0.16 \pm 0.26, p$ $=0.022$ ), but was normal at the time of diagnosis. Lymphocytes expressed low levels iNOS throughout the clinical course of acute KD (data not shown).

Table 3 shows a comparison of iNOS expression in neutrophils and monocytes in patients with and without CAL. In patients with CAL, iNOS expression in neutrophils was significantly higher only before treatment with IVGG $(p=0.011)$, and rapidly declined after treatment in most patients, irrespective of the presence of CAL (Table 3). However, the patients with CAL had higher monocyte-specific iNOS expression at 2 wk after onset $(p=0.035)$ (Table 3$)$, at the time that lesions were maximal.

Quantitation of circulating endothelial cells. Blood from controls contained a very small number of circulating endothelial cells, whereas blood from acute KD patients had significantly elevated numbers of circulating endothelial cells at the time of diagnosis (Fig. 2). The number of circulating endothelial cells continued to increase, even after IVGG administration, and peaked at $2 \mathrm{wk}(40.0 \pm 31.5 \mathrm{cells} / \mathrm{mL}$ versus $1.0 \pm 1.7$ cells $/ \mathrm{mL}, p<0.0001$ ) (Table 4). There were significantly more circulating endothelial cells in patients with CAL than those without CAL at both 2 and 3 wk after onset ( $p$ $=0.0119$ and $p=0.0151$, respectively), but the number of cells peaked in both groups at $2 \mathrm{wk}$. Most endothelial cells were positive for iNOS, but negative for eNOS (Fig. 2) and the number of iNOS-positive cells mirrored the number of endothelial cells (Table 4). Although we did not perform double staining with $\mathrm{P} 1 \mathrm{H} 12$ and iNOS, the morphology of iNOS positive endothelial cells was consistent with previously reported characteristics of circulating endothelial cells (19).

Table 3. iNOS expression of leukocytes in acute KD with or without $C A L$

With CAL ( $n$ 24) Without CAL (n 31) $\quad p$ Value

\begin{tabular}{lccc} 
Neutrophils (DMFI) & & & \\
Pretreatment & $1.51 \pm 0.86$ & $0.43 \pm 0.29$ & 0.011 \\
Post IVGG & $0.71 \pm 0.96$ & $0.49 \pm 0.46$ & $\mathrm{NS}$ \\
2 wk post-onset & $0.21 \pm 0.16$ & $0.15 \pm 0.24$ & $\mathrm{NS}$ \\
3 wk post-onset & $0.18 \pm 0.23$ & $0.09 \pm 0.16$ & $\mathrm{NS}$ \\
4 wk post-onset & $0.07 \pm 0.14$ & $0.12 \pm 0.16$ & $\mathrm{NS}$ \\
Monocytes (DMFI) & & & \\
Pretreatment & $0.04 \pm 0.03$ & $0.07 \pm 0.09$ & $\mathrm{NS}$ \\
Post IVGG & $0.05 \pm 0.02$ & $0.05 \pm 0.04$ & $\mathrm{NS}$ \\
2 wk post-onset & $0.78 \pm 0.39$ & $0.36 \pm 0.50$ & 0.035 \\
3 wk post-onset & $0.33 \pm 0.27$ & $0.27 \pm 0.39$ & $\mathrm{NS}$ \\
4 wk post-onset & $0.25 \pm 0.34$ & $0.15 \pm 0.13$ & $\mathrm{NS}$ \\
\hline
\end{tabular}

Data are mean \pm SD. $p$ Value indicates difference between groups. Table 4 . Quantitation of circulating endothelial cells in acute $K D$ with or without
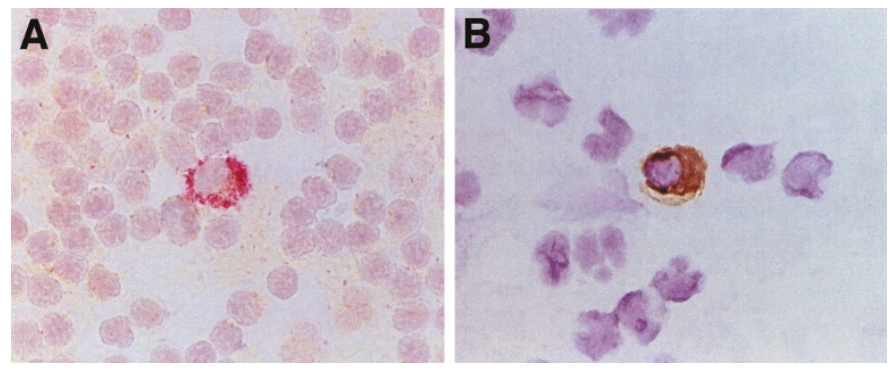

Figure 2. Immunohistochemical analysis of circulating endothelial cells. $(A)$ A buffy-coat smear stained with $\mathrm{P} 1 \mathrm{H} 12$ showing a circulating endothelial cell (stained red) (magnification $\times 1000)$. (B) A circulating endothelial cell stained for iNOS (stained brown) (magnification $\times 1000)$ in an acute KD patient with CAL.

Immunohistochemical findings in coronary artery in acute KD. In coronary artery specimens from each of the three patients with coronary aneurysms and sudden death (Table 2), severe panvasculitis with marked intimal thickening and destruction of the internal elastic lamina was observed. In these coronary arteries, endothelial cells were positive for iNOS rather than for eNOS. Massive infiltration of monocytes/ macrophages was noted in the intima and adventitia, and the majority of these cells demonstrated expression of iNOS. Furthermore, in dilated coronary artery with severe tissue damage and marked intimal thickening, most of the endothelial cells had dislodged from the arterial wall. In arterioles, the intima and adventitia were edematous and were infiltrated chiefly by iNOS positive monocytes/macrophages (Fig. 3). In coronary artery specimens from a normal control, eNOS expression was seen in endothelial cells, but iNOS expression was not seen in any cells (data not shown).

\section{DISCUSSION}

In acute $\mathrm{KD}$, various cytokines are released by activated monocytes/macrophages, including tumor necrosis factor- $\alpha$, IL-1, monocyte chemoattractant protein-1 (20), and VEGF (7). All these cytokines potentially induce iNOS expression in a wide variety of cells, including leukocytes, endothelial cells, and vascular smooth muscle cells (21). Our flow cytometric analysis clearly demonstrates that neutrophils, monocytes, and endothelial cells express iNOS at different stages in acute KD, suggesting their different roles in the development of CAL.

iNOS expression in neutrophils and tissue damage. $\mathrm{NOS}$ expression in neutrophils was maximal and restricted to the very early stage of acute $\mathrm{KD}$, especially in patients with CAL. This suggests that NO, synthesized by iNOS in neutrophils, may have a role in triggering the early tissue damage of CAL development in acute KD. iNOS expression results in the release of much more NO than eNOS expression in endothelial cells, and at high concentrations, NO is cytotoxic. Therefore, NO synthesized by iNOS may contribute to tissue damage and the early degenerative changes in the coronary artery wall (13, 14). In the very early stage of acute $\mathrm{KD}, \mathrm{WBC}$ counts, and particularly neutrophils, increase markedly (Table 1). Although we could not demonstrate the infiltration of neutrophils in the coronary artery wall, a previous pathologic study confirmed 
Table 4. Quantitation of circulating endothelial cells in acute KD with or without $C A L$

\begin{tabular}{|c|c|c|c|}
\hline & $\begin{array}{l}\text { With CAL } \\
(n=24)\end{array}$ & $\begin{array}{l}\text { Without CAL } \\
\quad(n=31)\end{array}$ & $p$ Value \\
\hline \multicolumn{4}{|c|}{ P1H12-positive cell number (cells/mL of whole blood) } \\
\hline Pretreatment & $2.4 \pm 4.3$ & $0.7 \pm 1.8$ & NS \\
\hline 2 wk post-onset & $55.5 \pm 31.8$ & $24.4 \pm 23.0$ & 0.011 \\
\hline 3 wk post-onset & $32.2 \pm 19.4$ & $11.3 \pm 10.0$ & 0.015 \\
\hline $4 \mathrm{wk}$ post-onset & $7.6 \pm 13.0$ & $3.7 \pm 5.5$ & NS \\
\hline Post IVGG & $4.4 \pm 2.2$ & $3.4 \pm 2.5$ & NS \\
\hline 2 wk post-onset & $20.9 \pm 8.0$ & $11.9 \pm 2.5$ & 0.012 \\
\hline 3 wk post-onset & $9.4 \pm 8.5$ & $4.6 \pm 3.5$ & 0.015 \\
\hline 4 wk post-onset & $0.6 \pm 1.0$ & $0.6 \pm 1.3$ & NS \\
\hline
\end{tabular}

Data are mean \pm SD. $p$ Value indicates difference between groups.

transient infiltration of neutrophils in the very early stage of acute KD, before infiltration of mononuclear cells (6). Recently, treatment with a neutrophil-elastase inhibitor, ulinastatin, was reported to be a potential adjunct to $\gamma$-globulin therapy in acute KD (22). iNOS expression in neutrophils may reflect their activation and enhanced function in the early phase of acute KD, as reported previously (23). These support the hypothesis that neutrophils contribute to NO production by enhanced iNOS expression in response to acute inflammatory stimuli and play an early restricted role in tissue damage in the coronary artery wall, without having a direct effect on the progression of CAL in acute $\mathrm{KD}$.

iNOS expression in coronary artery wall and circulating endothelial cells. Histopathological findings in acute KD show widespread vascular endothelial edema and necrosis, and leukocyte infiltration, involving coronary and other medium-sized muscular arteries, even in children without aneurysms detectable by echocardiography (4-6).

In normal vascular walls, eNOS is constitutively expressed by endothelial cells whereas iNOS is not (13). In the normal vascular wall, eNOS-derived $\mathrm{NO}$ works as a negative regulator in vascular remodeling in response to various stimuli, preventing leukocyte adhesion, platelet aggregation, and migration and proliferation of smooth muscle cells (24). In our study, iNOS was detected in the damaged coronary artery wall, whereas eNOS was not, suggesting up-regulation of iNOS expression and down-regulation of eNOS expression in the endothelial cells within KD-associated lesions. Furthermore, endothelial cells had dislodged from the coronary artery walls, showing marked intimal thickening and severe tissue damage (Fig. 3). Our findings suggest that endothelial damage and downregulation of the eNOS/NO pathway promote abnormal remodeling and facilitate pathologic changes in vessel wall morphology in acute KD.

During disease progression, the number of circulating endothelial cells and iNOS expression in these cells increased, particularly in patients with lesions, potentially correlating with the severity of tissue damage in the coronary artery wall. Increased numbers of circulating endothelial cells might be due to the physiologic displacement of cells from vessel walls by endothelial injury or to the expression of molecules such as thrombin that can cause the release of endothelial cells from the extracellular matrix. An earlier study also reported increased numbers of circulating activated endothelial cells in patients with sickle cell anemia, particularly at the onset of crisis (19). Circulating endothelial cells in acute KD tend to have an activated phenotype, as evidenced by enhanced expression of iNOS, and truly represent in situ endothelial cells. Increased levels of various cytokines including tumor necrosis factor- $\alpha$ and IL-1, activation of the coagulation system, abnormal adhesion of leukocytes to endothelium, and disturbances of vasoregulation are common findings in acute KD. All these factors affect the endothelial phenotype and may contribute to the development of CAL by modulating the expression of various hemostatic and adhesion molecules on endothelial cells. Although a pathophysiological role of iNOS immunoreactivity in circulating endothelial cells and in the endothelium of the coronary artery wall is still unclear, endothelium expressing iNOS in response to cytokines and anti-endothelial cell antibodies may interact with leukocytes and play a crucial role in the progression of CAL in acute $\mathrm{KD}$. The origin of iNOS circulating endothelial cells may be vascular wall or bone marrow. One limitation of this study is that we were unable to directly show that the increased number of circulating endothelial cells and expression of iNOS correlates with changes in the coronary arteries of these patients.

iNOS expression in monocytes/macrophages. We found an increased level of iNOS expression in patients with CAL, particularly in activated monocytes, at the time of CAL development. We also demonstrated massive infiltration of iNOSpositive monocytes/macrophages in the coronary artery wall in acute KD. It has been reported that the activation of monocytes/macrophages plays a central role in the development of vasculitis in acute KD (20). A previous histopathologic study demonstrated the infiltration of large numbers of mononuclear cells, such as lymphocytes and macrophages, into the vascular tissues of KD, implying that monocytes are recruited from the circulation in response to chemotactic stimuli (25). We have shown previously, using flow cytometric analysis, that VEGF expression in monocytes is maximal at $2 \mathrm{wk}$ post disease onset and is particularly high in patients with CAL (7). It is notable that the coronary artery lesions also develop at about $2 \mathrm{wk}$ after onset. We did not stain for VEGF in these patients, but recent studies demonstrated expression of VEGF by macrophages in 

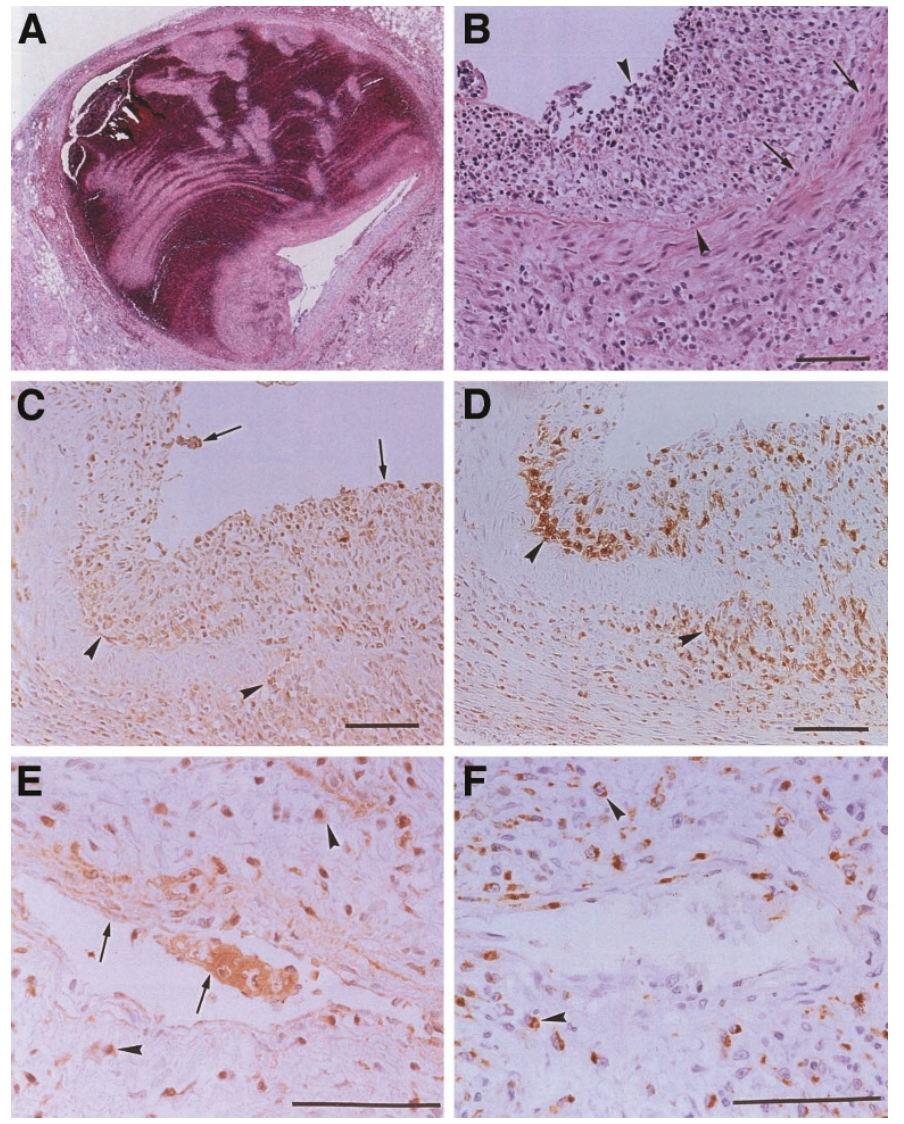

Figure 3. Immunohistochemical distribution of iNOS in the coronary artery from a patient with acute KD. The patient had multiple coronary aneurysms and died because of myocardial infarction on 18 of illness (patient 1 in Table 2). (A) An H\&E-stained section of the left anterior descending artery. Aneurysm formation with mural thrombosis is present and, in the wall of the aneurysm, severe panvasculitis with marked intimal thickening can be seen. (magnification $\times 15)(B)$ An H\&E-stained section of coronary arterial branch revealing vasculitis. Thickening of intima and disruption of the internal elastic lamina can be seen, with massive infiltration, chiefly by mononuclear cells and fibroblasts, in the intima and adventitia sparing the media (magnification $\times 150$; arrowheads: intima; arrows: disruption of the internal elastic lamina). (C) iNOS immunohistochemical staining of the coronary arterial branch. iNOS is detected in endothelial cells, infiltrating inflammatory cells, and fibroblasts in the intima and adventitia. Most of the endothelial cells were abnormal (magnification $\times 150$; arrowhead: macrophages; arrow: degenerated and desquamating endothelial cells). (D) CD68 immunohistochemical staining of the coronary arterial branch. Most of the infiltrating iNOS-positive cells observed in $C$ are confirmed to be monocytes/macrophages by the positive reaction for CD68 (magnification $\times 150$; arrowhead: macrophages). $(E)$ iNOS immunohistochemical staining of the arteriole. The intima and the adventitia are edematous and infiltrated by iNOS-positive cells (magnification $\times 300$; arrowheads: macrophages; arrow: disrupted endothelial cells). ( $F)$ CD68 immunohistochemical staining of the arteriole. iNOS-positive cells observed in $E$ are confirmed to be monocytes/macrophages by the positive reaction for CD68 (magnification $\times 150$; arrowheads: macrophages). Bars $=100 \mu \mathrm{m}$.

coronary artery tissue of patients with KD (26). Macrophagederived VEGF may induce chemotaxis of monocytes/ macrophages in both the inflammatory and remodeling processes, and may act in an autocrine or paracrine fashion in the coronary arterial walls of patients with acute KD. Further, activated macrophages, recruited to sites of damaged endothelium via VEGF, may participate actively in the inflammatory responses of the vascular walls by locally producing NO. In vitro studies have shown that VEGF increases NO release from human endothelial cells (9), and modulates microvascular permeability via a signaling cascade involving NO synthesis in coronary venules (10).

Study limitations. We could not provide immunohistochemistry of either uninvolved blood vessels from patients with $\mathrm{KD}$ and CAL or from patients with KD without CAL. Therefore, it is difficult to state whether the presence of iNOS protein is a cause or an effect of KD. NO has been shown to be beneficial in the setting of advanced atherosclerosis in transplant vasculopathy suggesting a cytoprotective role for iNOS (27). Further, Ozaki et al. (28) have recently shown that overexpression of eNOS accelerates atherosclerotic lesion formation in apoEdeficient mice. Both eNOS and iNOS have beneficial and also deteriorative characteristics in the vasculature. The environment in which NO is produced determines whether it is cytotoxic or cytoprotective. Expression of iNOS was demonstrated in coronary artery lesions even in the late phase of KD (A. Suzuki, personal communication), suggesting its role in active remodeling of damaged coronary artery. The potential functions of iNOS expression in KD remain unclear and may have both causative and protective roles in the vasculature at the different stages of KD.

\section{CONCLUSION}

The findings of our study suggest that NO is generated by neutrophils, monocytes, and endothelial cells dynamically at different stages of acute $\mathrm{KD}$, and correlates with the development of coronary artery lesions. Increased numbers of iNOSpositive circulating endothelial cells and enhanced iNOS expression in peripheral blood leukocytes appear to correlate with the severity of tissue damage in coronary artery wall in acute KD.

Acknowledgments. The authors thank Drs. Shunichi Fukuda, Seiichiro Takeshita, Keigo Nakaya, and Kunio Ohta for their helpful comments and suggestions. We also thank Drs. Shinichi Tsubata, Ayumi Miyazaki, Ken Suzaki, Masayoshi Miura, Gyokei Murakami, Masaharu Kasei, Daijiro Shima, Hiromichi Kubota, Kyoko Nakaaki, Youichi Onoue, Tadashi Noguchi, Chikako Sakai, and Hitoshi Moriuchi for their excellent assistance.

\section{REFERENCES}

1. Cuttica RJ 1997 Vasculitis, Kawasaki disease, and pseudovasculitis. Curr Opin Rheumatol 9:448-457

2. Suzuki A, Kamiya T, Arakaki Y, Kinoshita Y, Kimura K 1994 Fate of coronary arterial aneurysms in Kawasaki disease. Am J Cardiol 74:822-824

3. Kato H, Sugiyama T, Akagi T, Sato N, Hashino K, Maeno Y, Kazue T, Eto G, Yamakawa R 1996 Long-term consequences of Kawasaki disease. Circulation 94:1379-1385

4. Fujiwara T, Fujiwara H, Nakano H 1988 Pathological features of coronary arteries in children with Kawasaki disease in which coronary arterial aneurysm was absent at autopsy. Circulation 78:345-350

5. Fujiwara H, Hamashima Y 1978 Pathology of the heart in Kawasaki disease. Pediatrics 61:100-107

6. Naoe S, Shibuya K, Takahashi K, Wakayama M, Masuda H, Tanaka N 1991 Pathological observations concerning the cardiovascular lesions in Kawasaki disease. Cardiol Young 1:212-220

7. Brown TJ, Crawford SE, Cornwall ML, Garcia F, Shulman ST, Rowley AH 2001 CD8 T lymphocytes and macrophages infiltrate coronary artery aneurysms in acute Kawasaki disease. J Infect Dis 184:940-943 
8. Hamamichi Y, Ichida F, Xianyi Yu, Uese K, Tsubata S, Hashimoto I, Futatani T, Kanegane H, Miyawaki T 2001 Neutrophils and mononuclear cells express vascular endothelial growth factor in acute Kawasaki disease. Pediatr Res 49:74-80

9. Hood JD, Meininger CJ, Ziche M, Granger HJ 1998 VEGF upregulates ecNOS message, protein, and NO production in human endothelial cells. Am J Physio 274:H1054-H1058

10. Wu HM, Huang Q, Yuan Y, Granger HJ 1996 VEGF induces NO-dependent hyperpermeability in coronary venules. Am J Physiol 271:H2735-H2739

11. Anggard E 1994 Nitric oxide: mediator, murderer, and medicine. Lancet 343:1199 1206

12. Nathan C, Xie Q 1994 Nitric oxide synthase: roles, tolls, and controls. Cell 78:915918

13. Kibbe M, Billiar T, Tzeng E 1999 Inducible nitric oxide synthase and vascular injury. Cardiovasc Res 43:650-657

14. Beckman JS, Beckman TW, Chen J, Marshall PA, Freeman BA 1990 Apparen hydroxyl radical production by peroxynitrite: implications for endothelial injury from nitric oxide and superoxide. Proc Natl Acad Sci U S A 87:1620-1624

15. Geng Y, Hansson GK, Holme E 1992 Interferon- $\gamma$ and tumor necrosis facto synergize to induce nitric oxide production and inhibit mitochondrial respiration in vascular smooth muscle cells. Circ Res 71:1268-1276

16. Fukuda S, Hashimoto N, Naritomi H, Nagata I, Nozaki K, Kondo S, Kurino M, Kikuchi H 2000 Prevention of rat cerebral aneurysm formation by inhibition of nitric oxide synthase. Circulation 101:2532-2538

17. Research Committee on Kawasaki disease 1984 Report of subcommittee on standardization of diagnostic criteria and reporting of coronary artery lesions in Kawasak disease. Ministry of Health and Welfare, Tokyo

18. Iwai K, Miyawaki T, Takisawa T, Konno A, Ohta K, Yachie A, Seki H, Taniguchi N 1994 Differential expression of bcl-2 and susceptibility to anti-Fas-mediated cell death in peripheral blood lymphocytes, monocytes, and neutrophiles. Blood 84:1201-1208

19. Solovey A, Lin Y, Browne P, Choong S, Wayner E, Hebbel RP 1997 Circulating activated endothelial cells in sickle cell anemia. N Engl J Med 337:1584-1590
20. Terai M, Jibiki T, Harada A, Terashima Y, Yasukawa K, Tateno S, Hamada H, Oana S, Niimi H, Matsushima K 1999 Dramatic decrease of circulating levels of monocyte chemoattractant protein-1 in Kawasaki disease after gamma globulin treatment J Leukoc Biol 65:566-572

21. Kroncke KD, Fehsel K, Kolb-Bachofen V 1998 Inducible nitric oxide synthase in human diseases. Clin Exp Immunol 113:147-156

22. Zaitsu M, Hamasaki Y, Tashiro K, Matsuo M, Ichimaru T, Fujita I, Tasaki H, Miyazaki S 2000 Ulinastatin, an elastase inhibitor, inhibits the increased mRNA expression of prostaglandin H2 synthase-type 2 in Kawasaki disease. J Infect Dis. 181:1101-1109

23. Niwa Y, Sohmiya K 1984 Enhanced neutrophilic functions in mucocutaneous lymph node syndrome, with special reference to the possible role of increased oxygen intermediate generation in the pathogenesis of coronary thromboarteritis. J Pediatr 104:56-60

24. Rudic RD, Shesely EG, Maeda N, Smithies O, Segal SS, Sessa WC 1998 Direct evidence for the importance of endothelium-derived nitric oxide in vascular remodeling. J Clin Invest 101:731-736

25. Terai M, Kohno Y, Namba M, Umemiya T, Niwa K, Nakajima H, Mikawa A 1991 Class II major histocompatibility antigen expression on coronary arterial endothelium in a patient with Kawasaki disease. Hum Pathol 21:231-234

26. Suzuki A, Miyagawa-Tomita S, Komatsu K, Nishikawa T, Sakomura Y, Horie T, Nakazawa M 2000 Active remodeling of the coronary arterial lesions in the late phase of Kawasaki disease. Immunohistochemical study. Circulation 102:29352941

27. Shears II LL, Kawaharada N, Tzeng E, Billiar TR, Watkins SC, Kovesdi I, Lizonova A, Pham SM 1997 Inducible nitric oxide synthase suppresses the development of allograft arteriosclerosis. J Clin Invest 100:2035-42

28. Ozaki M, Kawashima S, Yamashita T, Hirase T, Namiki M, Inoue N, Hirata K, Yasui H, Sakurai H, Yoshida Y, Masada M, Yokoyama M 2002 Overexpression of endothelial nitric oxide synthase accelerates atherosclerotic lesion formation in apoE-deficient mice. J Clin Invest 110:331-340 